\title{
Big Bang Nucleosynthesis and the Origin and Evolution of Space-Time
}

\author{
Grant J. Mathews* \\ Center for Astrophysics, Department of Physics, University of Notre Dame, Notre Dame, IN \\ 46556, USA \\ E-mail: gmathewsand.edu
}

\section{T. Kajino}

Division of Theoretical Astronomy, National Astronomical Observatory of Japan, Mitaka, Tokyo 181-8588, Japan

Department of Astronomy, Graduate School of Science, University of Tokyo, Hongo, Bunkyo-ku, Tokyo 113-0033, Japan

E-mail: kajino@nao.ac.jp

\section{Yamazaki and M. Kusakabe}

Division of Theoretical Astronomy, National Astronomical Observatory of Japan, Mitaka, Tokyo 181-8588, Japan

E-mail: yamazaki.dai@nao.ac.jp, motohiko@kau.ac.kr

\section{M.-K. Cheoun}

Department of Physics, Soongsil University, Seoul 156-743, Korea

E-mail: cheounessu.ac.kr

Primordial nucleosynthesis remains as one of the pillars of modern cosmology. It is the testing ground upon which all cosmological models must ultimately rest. It is our only probe of the universe during the first few minutes of cosmic expansion and in particular during the important radiation-dominated epoch. There have been significant recent advances in the understanding of the big bang including the recent detection of the inflation-generated gravity-wave background. This talk will review the the current state of observational constraints on primordial abundances along with the key nuclear reactions, their uncertainties and how this knowledge places key constraints on cosmological models. In particular, we will summarize the connections between big bang nucleosynthesis and the birth of the universe, including cosmic gravity waves, inflation, the possible time variation of fundamental constants], the nature and origin of dark matter and dark energy, supersymmetry, and the primordial magnetic field.

XIII Nuclei in the Cosmos,

7-11 July, 2014

Debrecen, Hungary

\footnotetext{
*Speaker.
} 


\section{Introduction}

In recent years a clear picture of what the universe is comprised of, how long it has been in existence, and how it will evolve in the future has emerged. This knowledge derives from investigations via a number of cosmological probes including supernovae, observations of the large scale distribution of galaxies and the inter-galactic medium, analysis of the cosmic microwave background, and studies of the nucleosynthesis of the elements in the first few moments of cosmic expansion in the big bang along with the first stars of the early universe. Here, we review a number of remaining questions and highlight the input that big bang nucleosynthesis (BBN) provides toward answering them.

The best current understanding of the composition of the universe comes from the nine year data of the Wilkinson Microwave Anisotropy Probe WMAP [1] and the Planck Surveyor [2], combined with distance measurements from Type Ia supernovae [3], and a phenomenon referred to as Baryon Acoustic Oscillations [4]. We now know [1] that the fraction of the universe in the normal baryonic matter with which we are familiar is only $\Omega_{b}=0.0472 \pm 0.010$. A much larger fraction of the universe is made of a completely unknown component of "cold dark matter," $\Omega_{c}=$ $0.241 \pm 0.010 \pm 0: 016$. The universe is predominantly made of dark energy, $\Omega_{\Lambda}=0.712 \pm 0.010$. In addition to these, there is an almost negligible contribution from relativistic photons and neutri$\operatorname{nos} \Omega_{\gamma}=5 \times 10^{-5}$. It is a current dilemma that $95 \%$ of the universe involves forms of matter of which we know nothing about.

The precision with which these parameters are now known is much better than a decade ago, however, one should keep in mind that these parameters are based upon the simplest possible $\Lambda \mathrm{CDM}$ cosmology, and the analysis must be redone for more complicated cosmologies such as those described below. Also, besides the discrepancies between WMAP and Planck, there are still some discrepancies between the best fit cosmology and the observations. For example there is evidence of a suppression of the lowest multipole moments of the CMB. This may suggest $[6,7]$ evidence for a compact topology for the universe. There is also a debate in the literature $[8,9]$ as to whether the CMB dipole anisotropy extends to very large distances ( $\mathrm{Gpc}$ ) possibly corresponding to super-horizon pre-big bang curvature in the universe.

If the universe is homogeneous and isotropic, then the evolution of the early universe is simply given by the Friedmann equation describing the Hubble parameter $\mathrm{H}$ in terms of densities $\rho$, curvature $k$, the cosmological constant $\Lambda$, and the cosmic scale factor $a$ :

$$
\left(\frac{\dot{a}}{a}\right)^{2} \equiv H^{2}=\frac{8}{3} \pi G \rho-\frac{k}{a^{2}}+\frac{\Lambda}{3}=H_{0}^{2}\left[\frac{\Omega_{\gamma}}{a^{4}}+\frac{\Omega_{m}}{a^{3}}+\frac{\Omega_{k}}{a^{2}}+\Omega_{\Lambda}\right],
$$

where $H_{0}=69.33 \pm 0.88 \mathrm{~km} \mathrm{sec}^{-1} \mathrm{Mpc}^{-1}$ [1] is the present value of the Hubble parameter, and the various closure contributions from relativistic particles, nonrelativistic matter, curvature, and dark energy are defined respectively by $\Omega_{\gamma}=8 \pi G \rho_{\gamma} /\left(3 H_{0}^{2}\right), \Omega_{m}=8 \pi G \rho_{m} /\left(3 H_{0}^{2}\right), \Omega_{k}==k\left(a^{2} H_{0}^{2}\right)$, and $\Omega_{\Lambda}=\Lambda /\left(3 H_{0}^{2}\right)$.

When the scale factor was half of its present value, matter and dark energy contributed equally. When the universe was only $1 / 1200$ of its present size, relativistic photons and neutrinos dominated the universe. Indeed, this is the end of the radiation dominated "big bang". The early universe includes the Planck epoch, the birth of space-time, inflation, reheating, a variety of cosmic phase 
transitions (e.g. supersymmetry breaking, baryogenesis, the electroweak transition, and the QCD transition), the epoch of big bang nucleosynthesis (BBN), and the production of the cosmic microwave background (CMB). For most of the big bang only the radiation $\left[\Omega_{\gamma}\right.$ term in Eq. (1.1)] is important. There are, however, interesting variants of big bang cosmology where this is not the case. The probe used to support the big bang model as a whole is the spectrum of temperature fluctuations in the CMB which contains information of the first quantum fluctuations in the universe, and the details of the distribution and evolution of dark matter, baryonic matter and photons near the time of the surface of photon last scattering (about $3 \times 10^{5} \mathrm{yr}$ into the big bang). However, the only direct probe of the radiation dominated epoch is the yield of light elements from BBN in the temperature regime from $10^{8}$ to $10^{10} \mathrm{~K}$ and times of about 1 to $10^{3} \mathrm{sec}$.

\subsection{BBN reaction rates and light-element abundances}

The value of BBN $[10,11]$ as a cosmological constrain relies on the fact that the universe is in thermodynamic equilibrium at the relevant epoch. The temperature, density, and degrees of freedom here are all well constrained, and really the only parameters that matter for BBN. This is unlike other evolutionary phases of the universe, where other components control the observables. During BBN he only non-equilibrium processes of relevance are the nuclear reactions themselves which must be explicitly evolved through the BBN epoch. In all there are only 16 reactions of significance during BBN [11, 12]. Nevertheless, in order to be useful as a cosmological constraint one must know these rates to very high precision $(\sim 1 \%)$. Fortunately, unlike in stars, the energies at which these reactions occur in the early universe are directly accessible in laboratory experiments. Although considerable progress has been made [13,14] in determining the relevant rates, much better rates are still needed for the neutron life time [15, 16], the ${ }^{2} \mathrm{H}(p, \gamma)^{3} \mathrm{He},{ }^{2} \mathrm{H}(d, n)^{3} \mathrm{He}$, ${ }^{3} \mathrm{He}(d, p){ }^{3} \mathrm{He},{ }^{3} \mathrm{He}(\alpha, \gamma){ }^{7} \mathrm{Be}$, and ${ }^{7} \mathrm{Be}(n, \alpha){ }^{4} \mathrm{He}$ reactions.

One of the powers of standard-homogeneous BBN is that once the reaction rates are known, all of the light element abundances are determined in terms of a single parameter $\eta_{10}$ which is the baryon-to-photon ratio in units of $10^{10}$. The crucial test of the standard BBN is, therefore, whether a single value of $\eta_{10}$ can be found which reproduces all of the observed primordial abundances. There has been a recent thorough reviews [11], and also new constraints on the primordial helium abundance [17]. The best adopted abundance constraints are then as follows:

Based upon the spectra of narrow-line Lyman- $\alpha$ absorption systems, a conservative range for the primordial deuterium abundance of $\mathrm{D} / \mathrm{H}=\left(2.87_{-0.19}^{+0.22}\right) \times 10^{-5}$. This implies a $2 \sigma(95 \%$ C.L.) concordance region of: $2.49 \times 10^{-5}<D / H<3.3 \times 10^{-5}$ If one restricts the data to the six well resolved systems for which there are multiple Lyman- $\alpha$ lines[18, 19], one slightly lowers the deuterium constraint to $2.79 \times 10^{-5}<D / H<3.25 \times 10^{-5}$.

The abundance of ${ }^{3} \mathrm{He}$ is best measured in Galactic HII regions by the $8.665 \mathrm{GHz}$ hyperfine transition of ${ }^{3} \mathrm{He}^{+}$[20]. It is not yet understood, however, whether ${ }^{3} \mathrm{He}$ has increased or decreased through the course of stellar and galactic evolution [21, 22]. A best estimate [11, 5] is ${ }^{3} \mathrm{He} / \mathrm{H}$ $=(0.7 \pm 0.5) \times 10^{-5}$.

The primordial ${ }^{4} \mathrm{He}$ abundance, Yp, is best determined from HII regions in metal poor irregular galaxies extrapolated to zero metallicity. . In [17] it has been demonstrated that updated emissivities and the neutral hydrogen corrections generally increase the inferred abundance, while the correlated uncertainties increase the uncertainty in the final extracted helium abundance. The 
value and uncertainty from [?] of $Y_{p}=0.2465 \pm 0.0097$, is in general agreement with the predicted value from standard BBN when $\eta_{10}$ is fixed from the WMAP analysis [1].

The primordial abundance of ${ }^{7} \mathrm{Li}$ is best determined from old metal-poor halo stars at temperatures corresponding to the Spite plateau (see [11] and Refs. therein). There is, however, an uncertainty due to the the surface depletion of lithium in these stars. A reasonable upper limit on the ${ }^{7} \mathrm{Li}$ abundance is [11] $6.15 \times 10^{-10}$ based upon allowing for a possible depletion of up to a factor of $\sim 5$ down to the present observationally determined value $[23,24]$ of ${ }^{7} \mathrm{Li} / \mathrm{H}=1.58 \pm 0.35 \times 10^{-10}$ (95\% confidence limit). A lower limit can be taken from the $2 \sigma$ observational uncertainty in the presently observed value [11]. Recently we have made an exhaustive study of possible cosmological solutions to the ${ }^{7} \mathrm{Li}$ problem [25].

\section{WHAT ARE THE QUESTIONS?}

The important questions regarding the big bang are something like the following: 1) How did the universe begin? 2) Why are there 3 large dimensions? 3) What drives inflation? 4) Are there observable effects from: supersymmetric particles, string excitations, etc. 5) Is there evidence for large extra dimensions? 6) How does the universe reheat? 7) How and when was the net baryon number generated? 8) When and how was the dark matter generated? 9) When and how was the dark energy created? 10) Are there observable effects from the Electroweak or QCD transition? 11) Have the fundamental constants varied with time 12) Is there a primordial magnetic field?

Each of these questions can be probed by BBN. For example regarding items 1and 4, the birth of the universe out of the M-theory landscape can be associated with the production supersymmetric particles that can affect the reactions BBN (cf. [30]). Regarding item 11, the formation existence of extra dimensions can lead to time varying physical constants [31]. Regarding items 3, 6,7, 12 some scenarios for inflation, reheating and baryogenesis and associated magnetic field generation can lead to a modified expansion rate $[33,37]$. Similarly the early formation of dark matter and dark energy $[34,32]$ can alter the expansion rate. Any of such models that alter the expansion rate will affect the freezeout of the $\mathrm{n} / \mathrm{p}$ ratio and other nuclear reactions and hence, the will be constrained by their effect on light element abundances. We have recently investigated several of these questions in detail which we now briefly summarize.

Is there evidence of supersymmetric matter in the early universe? Although supersymmetry is a well motivated mathematical symmetry, it has been difficult to find evidence for its existence. Indeed, the lightest stable supersymmetric particle is still a candidate for the cold dark matter. If that is the case, then many other unstable supersymmetric particles would have been generated along with the dark matter in the very early universe. In particular, the next to lightest supersymmetric particle could have a lifetime long enough to be present and/or decay during BBN. If that is the case then the non-thermal decay of SUSY particles or the presence of a long-lived negatively charged stau particle (the supersymmetric partner of the tau) could lead to production of ${ }^{6} \mathrm{Li}$ during the early universe. The report [27] of enhanced ${ }^{6} \mathrm{Li}$ in metal poor stars appears [28] to be an artifact of 3D and non-local thermodynamic equilibrium (NLTE), the observed upper limit to the ${ }^{6} \mathrm{Li}\left(\sim 2 \%\right.$ of ${ }^{7} \mathrm{Li}$ abundance still places important constraints on supersymmetric models and there is still evidence [29] for primordial ${ }^{6} \mathrm{Li}$ in the SMC. At the same time ${ }^{7} \mathrm{Li}$ is as much as a factor of 3 below the BBN expectation. This might also be a manifestation of the existence of 
new unstable particles which decay during and/or after the big bang. In particular, in [30] we completed an exhaustive heavy negatively charged decaying $X^{-}$particles that modify BBN. The heavy $X^{-}$particles bind to the nuclei produced in BBN. The massive $X^{-}$particles reduce the reaction Coulomb barriers and enhance the thermonuclear reaction rates, extending the duration of BBN to lower temperatures. This can lead to a large enhancement of the ${ }^{6} \mathrm{Li}$ abundance [?] (for example by the ${ }^{4} \mathrm{He}_{X}\left(d, X^{-}\right)^{6} \mathrm{Li}$ reaction, while depleting ${ }^{7} \mathrm{Li}$. We find that $\mathrm{BBN}$ places a stringent limit on the lifetime of the $X^{-}$of $\tau_{X}=0.3-3 \times 10^{3} \mathrm{~s}$, far a mass of $1 \mathrm{TeV}$. Also the abundance per baryon must be $Y_{X}>0.04$.

Have the fundamental constants varied with time? A time dependence of fundamental constants in an expanding universe can be a generic result of theories which attempt to unify gravity and other interactions. In [35, 36] it was suggested that an increase in the average quark mass by an amount $\delta m_{q} / m_{q}=0.016 \pm 0.005$ provides a better fit observed light-element primordial abundances. In [31] we have carried out an independent evaluation of the effects on BBN from a variation in the parameter $\delta X_{q} / X_{q}$ motivated by new detailed analyses $[11,17]$ of the uncertainties in the observed light element abundance constraints. We made an independent evaluation of the resonant ${ }^{3} \mathrm{He}(d, p)^{4} \mathrm{He}$ reaction rate based upon both the forward and reverse reaction. We found that, although the uncertainty in the results increases due to variations in the resonance parameters, the revised abundance constraints narrow the range of possible variations in the quark mass from BBN. This latter constraint dominates and decreases the optimum concordance region to a value of $0.005<\delta m_{q} / m_{q}<0.007$. That is, the strong coupling constant cannot have varied by more than $0.7 \%$ by the epoch of BBN. Although some variation in the quark mass is not ruled out, the results of the present study are consistent with no variation in the averaged quark mass. Primordial abundances calculated in [31] as a function of variations in the quark mass $\delta m_{q} / m_{q}$ for a fixed value of $\eta$ deduced from the WMAP data.

Is there a primordial magnetic field? The existence of a primordial magnetic field (PMF) $\sim 1 \mathrm{nG}$ whose field lines collapse as structure forms is one possible explanation for the magnetic fields observed in galactic clusters. Such a PMF, however, could have influenced a variety of phenomena in the early universe such as the cosmic microwave background (CMB), (e.g. [37] and refs. therein). In [37] we reviewed correlations between the calculated CMB power spectrum (influenced by a PMF) and the primary curvature perturbations. We found that the PMF affects the CMB on both small and large angular scales in the TT and TE modes. The introduction of a PMF leads to a better fit to the CMB power spectrum for the higher multipoles, and the fit to the lowest multipoles can be used to constrain the correlation of the PMF with the density fluctuations. The best constraints on the PMF determined yet to date determined in our analyses are $|B|<2.10 \mathrm{nG}(68 \% \mathrm{CL}) ;<2.98 \mathrm{nG}(95 \% \mathrm{CL})$ on a present scale of $1 \mathrm{Mpc}$, and $n_{B}<1.19$ $(68 \% \mathrm{CL}) ;<0.25(95 \% \mathrm{CL})$ We found that the BB mode is dominated by the vector mode of the PMF for higher multipoles. We also showed that by fitting the complete power spectrum one can break the degeneracy between the PMF amplitude and its power spectral index.

Of particular relevance is that a primordial magnetic field can lead to fluctuations in the metric. The implied background in the induced gravity waves can then be used [37] to constrain the epoch at which the PMF was created. The balance between the expansion rate of the universe and various particle reaction rates has important effects on the nucleosynthesis of light elements in the bigbang. Moreover, since the energy density of the GWB contributes to the total energy density of 
the universe, the expansion rate is affected by the GWB. Therefore, on can indirectly constrain the energy density $\rho_{\mathrm{GW}}$ from the light element primordial abundances inferred from observations of deuterium (D), ${ }^{3} \mathrm{He},{ }^{4} \mathrm{He}$, and ${ }^{7} \mathrm{Li}$. Based upon the constraints on the parameters of the magnetic field one can conclude [37] that BBN most favors a magnetic field formed after the BBN epoch, although no earlier epoch is yet ruled out. It is hoped that an analysis based upon the newer high resolution date on smaller angular scales [2] will provide better limits.

Work at the University of Notre Dame was supported by the U.S. Department of Energy under Nuclear Theory Grant DE-FG02-95-ER40934.

\section{References}

[1] G. Hinshaw, et al. (WMAP Collaboration) ApJ, 208, 19 (2013).

[2] Planck Collaboration, PlanckúXVI, A \&A Submitted, arXiv:1311.1657v2 (2013).

[3] Sullivan, M., et al. 2011, ApJ, 737, 102; Suzuki, N., Rubin, D., Lidman, C., et al. 2012, ApJ, 746, 85

[4] N. Padmanabhan, et al., MNRAS, 427, 2132 (2012)

[5] A. Coc, this workshop proceedings (2014).

[6] M. Tegmark, A. de Oliveira-Costa and A. Hamilton, Phys. Rev., D68, 123523 (2003)

[7] D. Menzies, and G. J. Mathews, J. Cosmo. and Astropart. Phys. 10, p. 008 (2005).

[8] A. Kashlinsky, F. Atrio-Barandela, and H. Ebeling, Phys. Rep. (Submitted) (2012) arXiv:1202.0717

[9] G. J. Mathews, N. Q. Lan, and T. Kajino, Phys Rev. D, Submitted (2014).

[10] R. A. Malaney and G. J. Mathews, G. J., Phys. Rep. 229 , 145 (1993).

[11] F. Iocco, et al., Phys. Rep., 472, 1 (2009).

[12] R. H. Cyburt, Phys. Rev. D 70, 023505 (2004).

[13] P. Descouvemont, et al., ADNDT, 88, 203 (2004).

[14] R. H. Cyburt, et al., Astrophys. J. Suppl. 189, 240 (2010).

[15] A. P. Serebrov and A. K. Fomin, Phys. Rev. C 82, 035501) (2010).

[16] G. J. Mathews T. Kajino, and T. Shima, Phys. Rev. D71, 021302 (2005).

[17] E. Aver, K. A. Olive, and E. D. Skillman, JCAP 05 003, (2010), JCAP 11, 017 (2013).

[18] M. Pettini, et al. Mon. Not. Roy. Astron. Soc, 391, 1499 (2008); Pettini \& Cooke, MNRAS, 425, 2477 (2012).

[19] K. Olive, P. Petitjean, E. Vangioni and J. Silk, Mon. Not. R. Astron. Soc., 426, 1427 (2012).

[20] T. M. Bania, R. T. Rood and D. S. Balser, Nature 415, 54 (2002).

[21] C. Chiappini, A. Renda and F. Matteucci, Astron. Astrophys. 395, 789 (2002).

[22] E. Vangioni-Flam, K. A. Olive, B. D. Fields and M. Casse, Astrophys. J. 585, 611 (2003).

[23] S. G. Ryan, T. C. Beers, K. A. Olive, B. D. Fields and J. E. Norris, Astrophys. J. 530 , L57 (2000); S. G. Ryan, et al., Astrophys. J., 532, 654 (2000). 
[24] L. Sbordone, P. Bonifacio, E. Caffau et al., Astron. Astrophys., 522, 26 (2010).

[25] D. G. Yamazaki, M. Kusakabe,T. Kajino, G. J. Mathews and M.-K. Cheoun, Phys. Rev. D90, 023001 (2014).

[26] G. J. Mathews, K. Ichiki, T. Kajino, N. Q. Lan, Mod. Phys. Lett. A23, 1372 (2008).

[27] M. Asplund, D. L. Lambert, P. E. Nissen, F. Primas and V. V. Smith, Astrophys. J. 644, 229 (2006).

[28] Lind, K. et al., A\&A, 554, A96 (2013)

[29] J. C. Howk, N. Lehner, B. D. Fields, G. J. Mathews, Nature 489, 121123 (2012)

[30] M. Kusakabe, K. Kim, M.-K Cheoun, T. Kajino, Y. Kino, and G. J. Mathews, Astrophys. J. Suppl. 214, 5, (2014), arXiv:1403.4156.

[31] M.-K. Cheoun, T. Kajino M. Kusakabe and G. J. Mathews, Phys. Rev. D84, 043001,(2011).

[32] K. Umezu, K. Ichiki, T. Kajino, G. J. Mathews, R. Nakamura, Phys.Rev. D73, 063527 (2006).

[33] M. Yahiro, G. J. Mathews, K. Ichiki, T. Kajino, and M. Orito, Phys. Rev. D 65, 063502 (2002).

[34] K. Ichiki, M. Yahiro, T. Kajino, M. Orito, G. J. Mathews, Phys. Rev. D66, 043521 (2002).

[35] J. C. Berengut, V. V. Flambaum and D. F. Dmitriev, Phys. Lett. B683, 114 (2010)

[36] T. Dent, S. Stern and C. Wetterich, Phys. Rev. D76, 063513 (2007).

[37] D. G. Yamazaki, T. Kajino, G. J. Mathews, and K. Ichiki, Phys. Rep., 517, 141 (2012). 